\title{
Dolor abdominal agudo secundario a la rotura de tumor de Wilms: reporte de caso
}

\section{Acute Abdominal Pain Secondary to Wilms Tumor Rupture: Case Report}

\author{
1 Estudiante de Medicina, Facultad de Medicina, Universidad Nacional \\ de Trujillo, Trujillo, Perú \\ 2 Especialista en Urología, Magíster en Investigación y Docencia \\ Universitaria, Universidad Nacional de Trujillo, Departamento de \\ Urología, Hospital Regional Docente de Trujillo, Trujillo, Perú
}

Jose Luis Lecca-Zavaleta ${ }^{1}$ Segundo Victor Salirrosas Bermudez, MD, $\mathrm{Mg}^{2}$

Urol Colomb 2020;29:178.

\section{Retraction Notice}

"Concerning the article "Dolor abdominal agudo secundario a la rotura de tumor de Wilms: reporte de caso" by LeccaZavaleta, J.L. \& Bermudez, S.V.S.

The editors were made aware of a previously published article in Revista Médica de Trujillo (2017;12(3):134-8) with the same title, authors and content.

According with the international committee of medical journal editors (ICMJE):

"Authors who attempt duplicate publication without such notification should expect at least prompt rejection of the submitted manuscript. If the editor was not aware of the violations and the article has already been published, then the article might warrant retraction with or without the author's explanation or approval"

For these reasons Revista Urologia Colombiana gives notice that the paper is retracted by the Editor-in-Chief and the Editorial Committee in accordance with COPE guidelines."

\section{Aviso de Retractación}

"Sobre el artículo“ Dolor abdominal agudo secundario a la rotura de tumor de Wilms: reporte de caso "de LeccaZavaleta, J.L. \& Bermudez, S.V.S ,.

Los editores tuvieron conocimiento de un artículo publicado previamente en Revista Médica de Trujillo (2017; 12 (3): 134-8) con el mismo título, autores y contenido.

Según el comité internacional de editores de revistas médicas (ICMJE, por sus siglas en inglés):
Address for correspondence Jose Luis Lecca Zavaleta, Las Lomas Mz. 29 Lt. 34 Huanchaco. Trujillo, Perú (e-mail: jlek_2511@ @hotmail.com).

"Los autores que intentan la publicación duplicada sin notificación deben esperar al menos un rechazo inmediato del manuscrito enviado. Si el editor no estaba al tanto de las violaciones y el artículo ya se publicó, entonces el artículo podría justificar la retractación con o sin la explicación o aprobación del autor"

Por estas razones, la Revista Urologia Colombiana notifica que el Editor en Jefe y el Comité Editorial se retracta del artículo de acuerdo con los lineamientos COPE ".

\section{Referencias}

1 Lonergan GJ, Martínez-León MI, Agrons GA, Montemarano H, Suarez ES. Nephrogenic rests, nephroblastomatosis, and associated lesions of the kidney. Radiographics 1998;18(04):947-968

2 PDQ Pediatric Treatment Editorial Board. Wilms Tumor and Other Childhood Kidney Tumors Treatment (PDQ $®)$ : Health Professional Version. 2017 Jun 14. In: PDQ Cancer Information Summaries [Internet]. Bethesda (MD): National Cancer Institute (US); 2002-. Available from: https://www.ncbi.nlm.nih.gov/books/NBK65842/

3 Godziński J, Weirich A, Tournade MF, y col. Primary nephrectomy for emergency: a rare event in the International Society of Paediatric Oncology Nephroblastoma Trial and Study no. 9. Eur J Pediatr Surg 2001;11(01):36-39

4 Al-Hussain T, Ali A, Akhtar M; Al-HussainT. Wilms tumor: an update. Adv Anat Pathol 2014;21(03):166-173

5 Davidoff AM. Wilms tumor. Adv Pediatr 2012;59(01):247-267

6 Basak E. Wilms Tumor and Its Management in a Surgical Aspect. In: Marry M, van den Heuvel-Eibrink, editors. Wilms Tumor. Brisbane (AU): Codon Publications; 2016. Chapter 4. Available from: https://www.ncbi.nlm.nih.gov/books/NBK373361/

7 Kosloske AM, Mclver WJ, Duncan MH. Intraperitoneal rupture of a Wilms' tumor. West J Med 1985;142(05):694-696
DOI https://doi.org/

$10.1055 / \mathrm{s}-0038-$

$165151110.1055 / \mathrm{s}-0038-$

1651511.

ISSN 0120-789X0120-789X.

eISSN 2027-0119.
Copyright $\odot 2020$, Sociedad Colombiana License terms de Urología. Publicado por Thieme Revinter Publicações LtdaSociedad Colombiana de Urología. Publicado por Thieme Revinter Publicações Ltda., Rio de Janeiro, Brazil. Todos los derechos reservados.

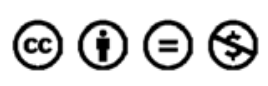

